\title{
COLLABORATIVE WEB GIS PLATFORM FOR SYSTEMATIC EXPLORATION OF LAKE GENEVA
}

\author{
Yosef Akhtman, Lorenzo Martelletti, Olga Grandjean and Ulrich Lemmin \\ TOPO Lab, École Polytechnique Fédérale de Lausanne, Switzerland \\ \{yosef.akhtman,lorenzo.martelletti,olga.grandjean,ulrich.lemmin\}@epfl.ch \\ topo.epfl.ch, www.elemo.ch
}

KEY WORDS: GIS, Mapping, Surveying, Visualisation, Navigation, Web-GIS, Database, Underwater

\begin{abstract}
:
We have developed and deployed a Web-based GIS data management framework, which facilitates an effective and highly structured search, retrieval and visualisation of multi-modal scientific data, as well as its subsequent dissemination in multiple and standardised forms beneficial for both the research partners involved in the project and the general public. In the context of the long term objectives of the Élémo project, the developed methodology may be utilised for automated and systematic collection of the multifaceted scientific data and with the goal of assembling a comprehensive database encompassing all aspects of currently planned and future scientific investigations.
\end{abstract}

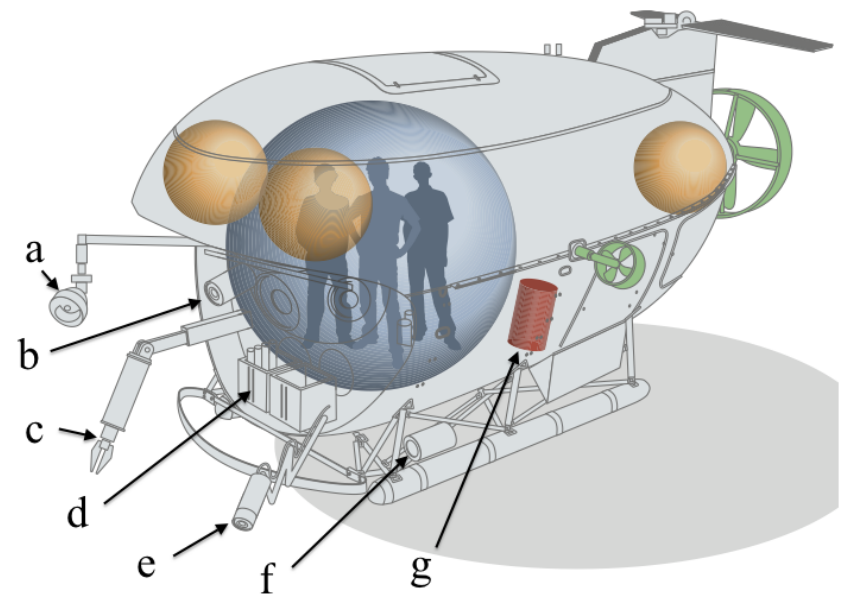

Figure 1: General structure of MIR 1 and 2 submersibles (Adopted from www.elemo.ch). Examples of onboard instrumentation included (a) projectors; (b) 3D camera system; (c) articulated arm; (d) sample storage; (e) mobile camera; (f) water sampling device; (g) particle filters.

\section{INTRODUCTION}

The Élémo project (www.elemo.ch) constitutes a multidisciplinary collaborative research initiative involving two deep-water submersibles operated by the P.P. Shirshov Institute of Oceanography of the Russian Academy of Science, as well as ten leading Swiss and international research institutions. The objectives of the project include a comprehensive "source-to-sink" investigation of the multiple aspects of the lake Geneva environmental functioning encompassing its geology, chemistry, biology and physics.

The initial stage of the Élémo project involved a period of intensive field work during the summer months of 2011, including the total of 30 diving days for each of the two MIR submersibles operated by the Russian collaborators. In the course of the initial field campaign a wide range of state-of-the-art scientific equipment attached to the MIR submersibles illustrated in Figure 1 has been utilised to collect a comprehensive array of environmental, navigational, as well as dense sensory data. The instruments employed included among others multiple extended CTD analysers, two advanced in-situ mass spectrometers ${ }^{1}$ and a voltammetric profiling system ${ }^{2}$. Both discrete and continuous sampling of water and lake-floor sediment has been carried out using a Rosette sampling system ${ }^{2}$, Niskin bottles, particle filters $^{3}$, as well as a sediment coring system ${ }^{4}$. The physical properties of the lake-floor sediment have been probed using Nimrod and cone penetrometers ${ }^{4}$. The navigation and environmental data has been further acquired using a 3D imaging system, Doppler velocity $\operatorname{logs}$ and a multi-beam sonar ${ }^{5}$, as well as an onboard articulated camera and an augmented GPS-based navigation system ${ }^{6}$.

The initial field campaign has been carried around the three representative regions of Lake Geneva, as illustrated in Figure 2. Specifically, the Vidy bay represents a densely populated coastal zone, where the outfall of the Lausanne sewage treatment plant brings water masses and pollutants into the lake. The Rhone river delta constitutes a major source of water and sediment. While, the deep water zone in the centre of the lake act as the "sink" for the various materials and pollutants that introduced into the lake along its coastal line.

From a scientific point of view, the project presents a unique opportunity for cross-disciplinary and inter-institutional collaboration. This opportunity, however, is accompanied by a major challenge associated with the development of both effective and efficient methodology for management, sharing and dissemination of diverse, multimodal, and often poorly synchronised scientific data(Dragicevic, 2004, Fu and Sun, 2010).

The rest of this paper is constructed as follows. In Section 2 we discuss three major stages in the data organisation process. Namely, we detail the consolidation of the master navigation data in Section 2.1, we describe the process of indexing of the video logs in

\footnotetext{
${ }^{1}$ Richard Camilli, et al., Woods Hole Oceanographic Institution, USA.

${ }^{2}$ Marylou Tercier-Waeber, Jean-Luc Loizeau, et al., University of Geneva, Switzerland.

${ }^{3}$ Samuel Aray, et al., EPFL.

${ }^{4}$ Ulrich Lemmin and Nicolas Le Dantec, EPFL, Switzerland.

${ }^{5}$ Oscar Pizaro et al., University of Sydney, Australia; Angelos Mallios, University of Gerona, Spain.

${ }^{6}$ Anatoly Sagalevich, Shirshov Institute of Oceanography, Russia.
} 


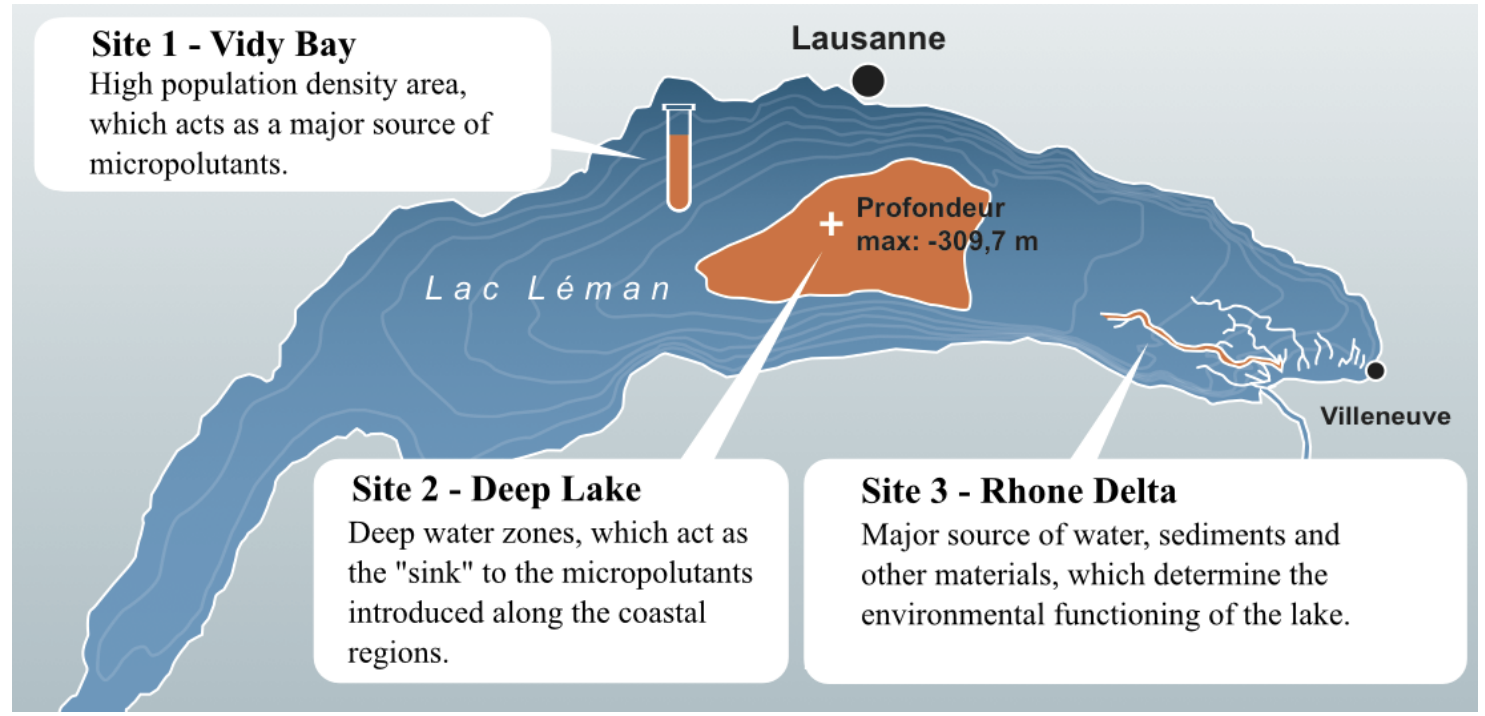

Figure 2: Three representative regions surveyed in the course of the initial stage of the Élémo project (Adopted from www .elemo.ch).

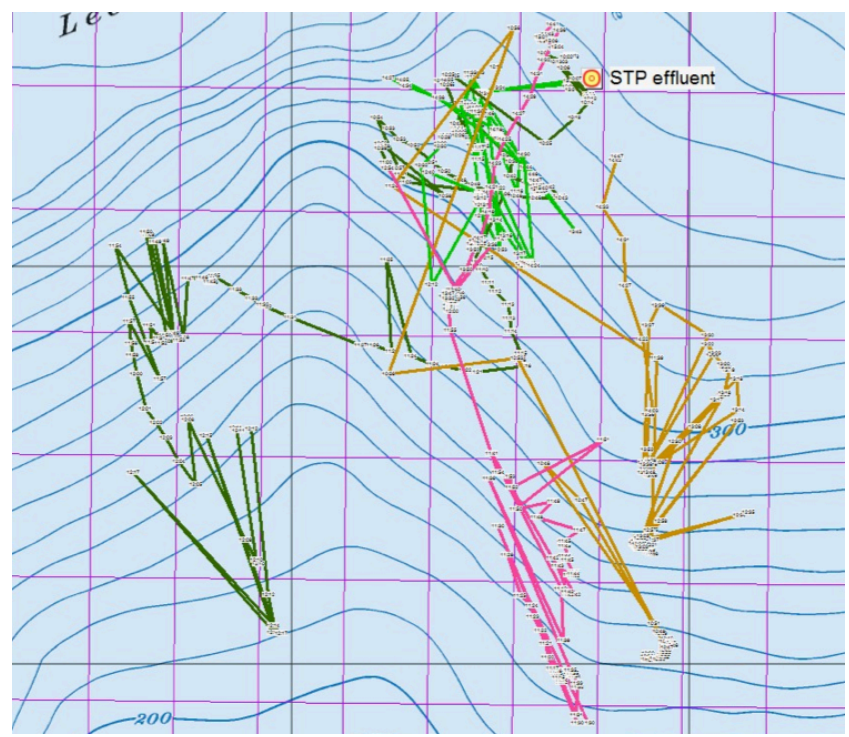

Figure 3: Underwater trajectories of the MIR submersibles in the Bay of Vidy obtained from the raw position data recorded by the surface navigation system (Courtesy of J.-L. Loizeau).

Section 2.2 and finally outline the structure of the developed WebGIS platform in Section 2.4 before drawing our conclusions in Section 3.

\section{METHODOLOGY}

\subsection{Master navigation data}

The first major challenge that we have encountered in our effort to construct a comprehensive representation of the collected data was the reliable estimation of the underwater trajectories of the submersibles Mir 1 and 2 throughout the course of the field campaign. Evidently, an accurate position is essential for the correct interpretation of the collected environmental data. Unfortunately, the position information recorded by the submarines' navigation system was largely unreliable and had many obvious outliers. The raw

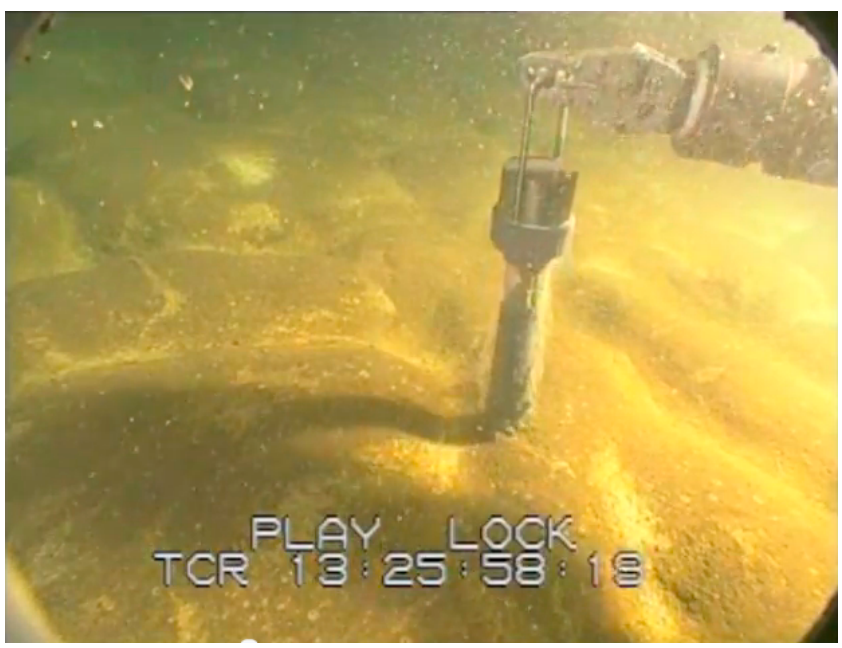

Figure 4: A typical frame from the video log enquired by the main camera of the MIR submersible in the course of the field campaign in lake Geneva.

trajectories of the submersibles in the Vidy bay are portrayed in Figure 3.

Correspondingly, the navigation data describing the 3D trajectories of the MIR submersibles has been comprehensively analysed and consolidated. In particular, the reliability of the navigation data has been improved significantly by the means of suppression of instantaneous outliers, as well as by non-linear interpolation of missing GNSS data. The submersible navigation trajectories become particularly informative in the presence of high-resolution bathymetry data, which is presently available for a considerable portion of the dive campaign area.

\subsection{Indexing of the video logs}

The objective of video processing was twofold: to prepare daily video clips suited for online streaming and to synchronise such videos with navigation data. Video data was originally provided as a set of 24 disk image files (68.04 GB) covering 50 daily missions 
(a)

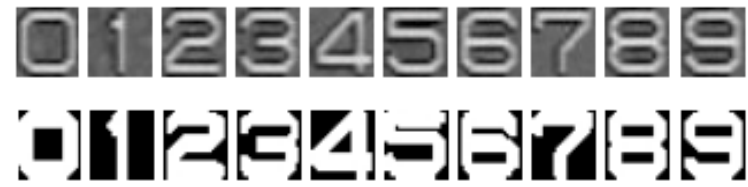

Figure 5: Digits (a) and masks (b) utilised by the digit classification algorithm.

(26 from Mir 1 and 24 from Mir 2) for a total of $27 \mathrm{~h} 43$ of video recording. We processed each disk image using Apple's iMovie software, extracting 50 audio/video quicktime files. These clips were eventually uploaded to YouTube, as we considered it the most practical and sophisticated tool to stream video for the project's scope. In order to build the time reference string of each clip, given the length of video data, we decided to use an Optical Character Recognition (OCR) approach over video frames. Quicktime files were further processed to obtain image sequences with a single frame-per-second frequency, concordant with navigation data frequency. OCR was carried out with a C++ script using OpenCV 2.3 (Open Source Computer Vision), a library of programming functions for real time computer vision. The idea was to estimate each video frame digit by comparing it with a known set of digits in the form of an optimisation problem. Initially, from selected images we created a sample set of 10 greyscale digits and a corresponding set of 10 black and white digit masks (resp. pixel values 0 and 1). Through code routines we compared the image's region of interest corresponding to each one of the 6 time digits (hh:mm:ss) with the sample set of digits and masks portrayed in Figure 5 (a) and (b) respectively. Subsequently, the estimate of each desired digit is obtained as a solution of an optimisation problem, which may be formulated as follows

$$
\hat{d}=\underset{i=0 \ldots 9}{\arg \min }\left(\frac{1}{\left\|\boldsymbol{M}_{i}\right\|_{L_{1}}}\left\|\left(\boldsymbol{R}-\boldsymbol{D}_{i}\right) \circ \boldsymbol{M}_{i}\right\|_{L_{1}}^{2}\right),
$$

where $\circ$ denotes the element-wise, or Hadamard product, $\boldsymbol{R}$ represents a $24 \times 26$ pixel region of interest extracted from the original image exemplified in Figure 4, while $\boldsymbol{D}_{i}$ and $\boldsymbol{M}_{i}$ are the $i$-th digit and mask, as depicted in Figure 5 (a) and (b) respectively. The minimum result provides a numeric digit corresponding to the one represented in the original video frame. Our code output a csv (Comma Separated Values) file containing a numeric sequence of digital timestamps. Due to strong changes in video frames background, which was varying from pale yellow to dark green, the results were affected by errors. Hence, we used Excel and ad-hoc algorithms to check for errors and correct them. The final result was a time indexing string, like the one reported in the following example, composed by substrings of initial time, ending time and total seconds from the beginning, one for each continuous video sequence inside a single mission video.

The resultant video index string is illustrated in Table 2 lines 33-36. Using this strings, we were finally able to index videos on YouTube and to synchronise them exactly with navigation data.

\subsection{Existing WebGIS solutions}

While developing our project-specific WebGIS platform, we have carried out a comprehensive review of the Web-based GIS solutions currently available. Specifically, the key features we were looking for were the capability to manage dense time and space 4D datasets, to be collaborative (i.e. multiple users being able to input and manage datasets), to be client-side, and to be easily configurable and extensible. In particular, the application frameworks that have been identified as potentially satisfying our requirements are MapFish (www.mapfish.org), Geomajas (www.geomajas.org), and MapBender (www . mapbender . org).

MapFish - A project of the Open Source Geospatial Foundation (OSGeo Foundation, www . osgeo . org) that provides an open source web mapping development framework that extends the Pylons Python development framework with geospatial-specific functionality and uses the Javascript libraries OpenLayers, ExtJS and GeoExt.

Geomajas - An open source webGIS framework with client-server interaction that allows powerful displaying and editing of spatial data. Like MapFish, Geomajas is an OSGeo project and is compliant with Open Geospatial Consortium (OGC, www . opengeospatial org) standards (WMS, WFS, GML). Geomajas has a strong server side focus where processing, styling, filtering, caching, etc. of geospatial data happens, while a powerful data management and visualisation interface is available for multiple users through their browsers. Moreover, similarly to our approach, Geomajas is designed with a plugin structure that allows extensibility and adaptability.

MapBender - A back office software and client framework for spatial data infrastructures. The software is implemented in PHP, JavaScript and XML and dual licensed under GNU GPL and Simplified BSD license. It provides a data model and web based interfaces for displaying, navigating and querying OGC compliant map services. There are many installations of MapBender, especially in Germany, some of which utilise the features of the jQuery JavaScript framework (jquery.com) and displaying data profiles coupled with planar mapping.

Subsequently, we have concluded that although theoretically possible, none of the considered frameworks has demonstrated the capability to facilitate the visualisation of synchronised multi-dimensional data. Our dataset, like many coming from marine and aerial surveys, has strong need of space and time data correlation. We have therefor decided to develop a simple but powerful system to cater for our specific usage scenario. Furthermore, we could not identify an application which would provide a client-side implementation. Client browser are becoming extremely powerful, as well as HTML and Javascript languages. Moreover this feature allows a webGIS system to run on mobile devices and be configured as mobileGIS during surveying. We have concluded that this is a core requirement in our field of application.

\subsection{Developed Web-GIS platform}

Correspondingly, the following items (a)-(k) provide a brief description of the Data Browser visual components as indicated in Figure $6(\mathrm{a}-\mathrm{k})$, respectively.

a) 2D Map - The central part of the data browser interface portrayed in Figure 6 is constituted by a Google maps-based interactive map, which provides the geographical references of the collected data. Google maps API was used to support the spatial part of the survey's visualisation and cover the first two dimensions of the data. The underwater trajectories of both submersibles Mir 1 and 2 are colour-coded in accordance to their correspondence to either of the submersibles (red and blue for Mir 1 and 2 respectively). When a trajectory is selected in the interface, related information is displayed in the other parts of the page to display the most complete overview possible of different aspects of this dive. Discrete samples and observations are indicated with markers, as described in the Visualisation Key portrayed in in Figure 6(b). 

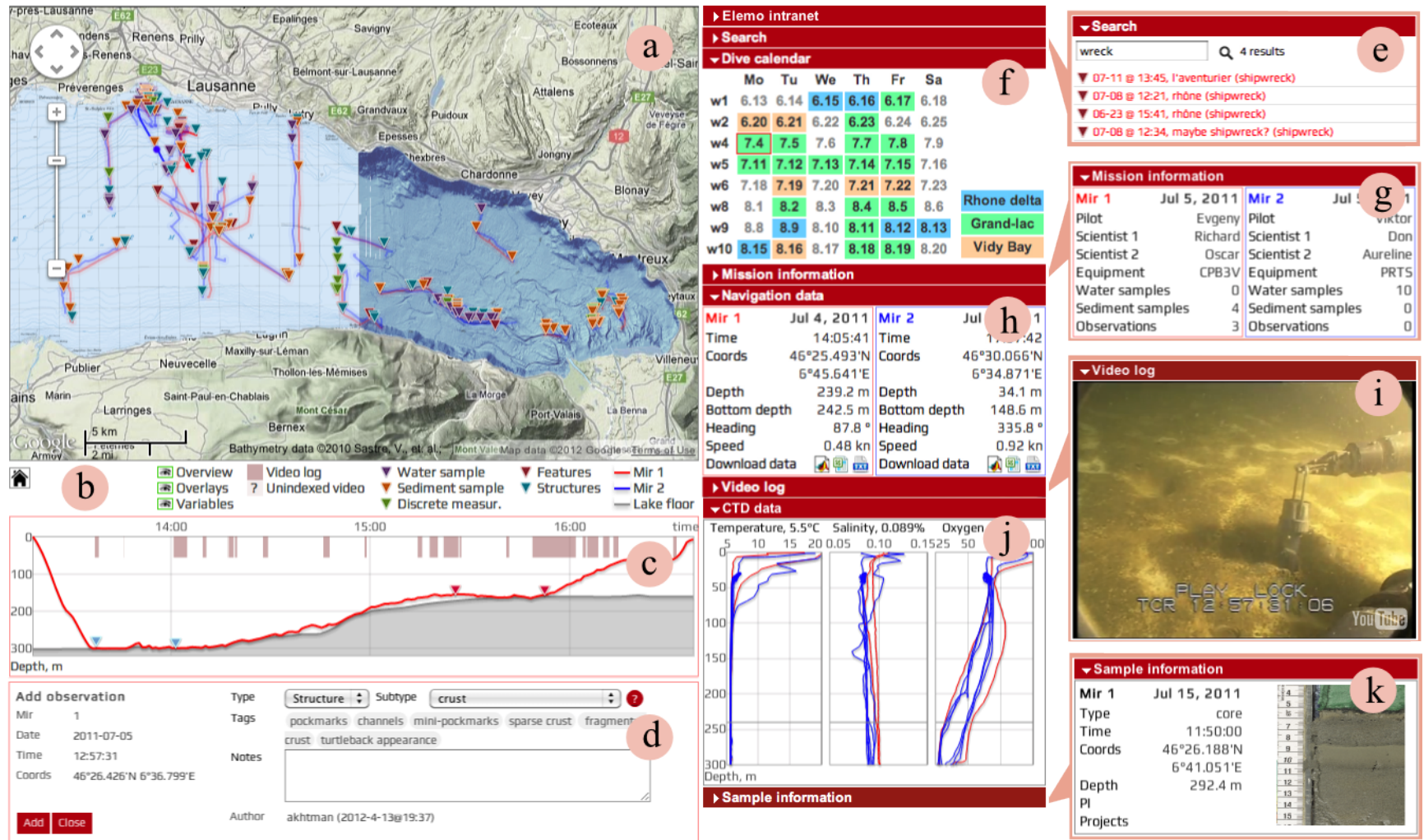

Figure 6: Main components of the current implementation of the Élémo project data browser: (a) Google maps-based two-dimensional interactive map; (b) visualisation key; (c) timeline with depth versus time dive profile; (d) observation entry/editing form; (e) search form; (f) field campaign dive calendar; (g) mission information (h) navigation data; (i) video log; (j) master environmental variables (temperature, salinity and oxygen); (k) sample information (sediment analysis courtesy of Corella et al.).

b) Visualisation Key - A detailed description of the various visualisation elements across multiple visualisation panels, in particular the main map and the timeline chart depicted in Figure 6(a) and (c), respectively. In addition to its role of legend, the key panel facilitates control over visibility of the various visualisation elements. For example, the visibility of the various types of discrete sample may be toggled by clicking on the corresponding marker in the legend. Furthermore, the legend provides a mechanism for controlling the visibility of map overlays and sensor data visualisations. For instance, the map depicted in Figure 6(b) combines the basic Terrain model of Google maps with the bathymetry data provided by the SwissTopo National map (www. swisstopo.ch), as well as the high-resolution bathymetry data (Sastre et al. 2010).

c) Timeline - A profile of depth of each submarine during the time span of the dive was created to complete the missing dimension of mapping. This plot depicts the depth of the submarine and of the bottom of the lake versus the time sequence of the dive. Samples and observations are also represented. Synchronisation mechanisms link real-time hovering of these plots with others visualisations tools (like position of the map and sensory data).

d) Observation form - The observation form facilitates collaborative analysis of the available video logs. In particular the classification of the various types of lake floor morphology is carried out by systematic review of the video logs and the periodic addition of observations whenever a reliable classification of the floor morphology on the video frame is possible.

e) Search - The search feature consists of a simple input text box and a retractable area for text results. The search is performed through observations and samples, but it is theoretically extendible to any kind of data. The use of jQuery library and the logic of including searchable visualisable data in the Document Object Model (DOM) structure, allow for a markedly fast live search. Once search results are retrieved, the plugin displays the results on the list and triggers a highlight event. The map feature listens to this event and displays only markers related to search results. As an example of the usage of this feature, the Search plugin allows to visualise in one click all instances where wrecks have been observed in videos, indicating the dive trajectory and displaying wrecks on the map of the Lake.

f) Calendar - The calendar component depicted in Figure 6(e) facilitates fast access to data collected on any particular date of the field campaign. The dates on the calendar are colour-coded according to one of the three regions of interest (Vidy Bay, Rhone Delta and Deep Lake) investigated on that day. The calendar component provides an intuitive interaction with the browser through standard mouse gestures, such as "mouseover" and "click". For example the "mouseover" results in the corresponding trajectories on the main map to be highlighted, as well as the corresponding mission information to be displayed in the "mission information" component of the data browser UI.

g) Mission Information - The mission-specific information including the names of the pilot and the scientists on board, as well as the list of operating instruments and the number of collected samples is displayed in the corresponding panel of the data browser. The mission information is updated whenever a new trajectory is selected using the "mouseover", or "click" mouse gestures. 
h) Navigation Information - The detailed navigation information including the geographical position, depth, depth of the lake floor, as well as heading and speed are indicated in the navigation panel of the data browser. The navigation information remains synchronised with other visualisation panels and is updated in accordance to the state of browser in both interactive and replay modes.

i) Video Log - The availability of a video $\log$ is indicated on the timeline chart depicted in Figure 6(c). Correspondingly, the replay of the available video sequences may be triggered "clicking" the corresponding area of the timeline chart. During replay, all visualisation components, including the map, the timeline, as well as the navigation information remain synchronised with the particular frame of the video sequence. Clicking the video reveals an Observation form depicted in Figure 6(c), which facilitates collaborative aggregation of observations.

j) Master Environmental Variables - Sensory data of water quality have been monitored during all dives, registering temperature (C), oxygen level (\%) and salinity (\%) of the water. Irregular frequency of measurements was unified by interpolating all data (how) to one second time-step Theses data are each plotted versus depth. If there is no selected day, all the dataset is represented, allowing an overall view of behaviour of theses variables. On the other hand, if a specific day is selected theses plots each show one line per submarines color-wise represented for this daily data. A crosshair bar is synchronised with the browsing of the depth plot.

k) Sample Information - If a sample icon is clicked on the map or on the timeline, information about this sample is displayed (type, time, coordinates, coordinates, people that collected it and project it was used on). Actual content of the sample are not yet available online on this platform.

\subsection{Data structure}

The developed GIS platform includes a Google Fusion Tables-powered online database of temporally and spatially synchronised sensory data, which allows systematic search, retrieval, as well as collaborative editing. In particular, the semantic structure of the entire dataset is depicted in Figure 7. The resultant data is stored using XML, specifically the Open Geospacial Consortium (OGC) KML and OGC GML/Observations and Measurements $(\mathrm{OM})+$ Sensor Web Enablement (SWE) schemas as detailed in the corresponding OGC standards. The simplified examples of the XML files that are utilised by our Web GIS platform are depicted in Tables 1 and 2 .

\section{CONCLUSIONS}

We have developed a collaborative Web-based GIS platform, which facilitates efficient management, sharing and dissemination of multimodal scientific data.

Multiple sources of multi-modal data, including continuous in-situ measurements, as well as discrete water and sediment samples have have been accurately geo-referenced and mapped. The precise synchronisation of the video logs collected throughout the entire course of the field campaign has been achieved by the frame-by-frame timestamp analysis. This allows for a classification of the lake floor morphology; analysis of the sediment mechanical properties; as well as observation and classification of the lake flora and fauna. The developed Web-GIS platform provides the necessary collaborative functionality to facilitate such analysis.

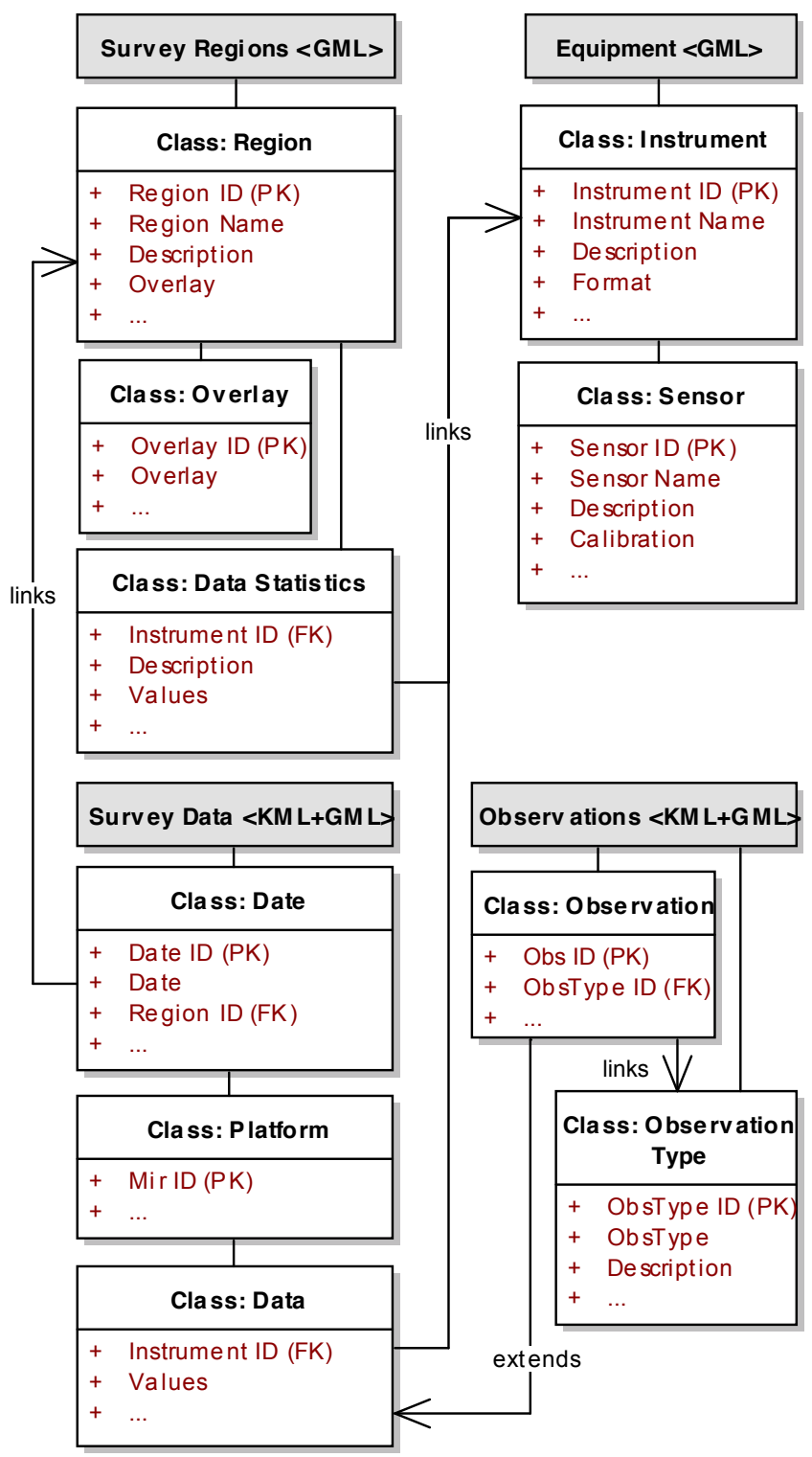

Figure 7: Semantic diagram of the Élémo project dataset.

A modular version of the developed platform is currently under development. The aim of the new structure is to create a separate independent plugin for each of the visualisation components, which are implemented as independent plugins, which interact between each other by the mean of asynchronous events. This solution would achieve the goal of reusability and modularity. New plugins can be developed easily for the use of different specialised scientific investigations.

The public version of the developed GIS platform is available online at: http://elemo-research.epfl.ch/demo.html.

\section{ACKNOWLEDGEMENTS}

The authors would like to thankfully acknowledge the support of Ferring Pharmaceuticals and the Russian Federations Honorary Consulate in Lausanne, as well as the devoted crew of P.P. Shirshov Institute of Oceanography of the Russian Academy of Science for facilitating this research. 
Table 1: Description of a single day collected in the course of Élémo field campaign and encoded using a combined OGC KML (2008)/GML (2012) format as suggested by Lake (2007). The corresponding GML content is detailed in Table 2.

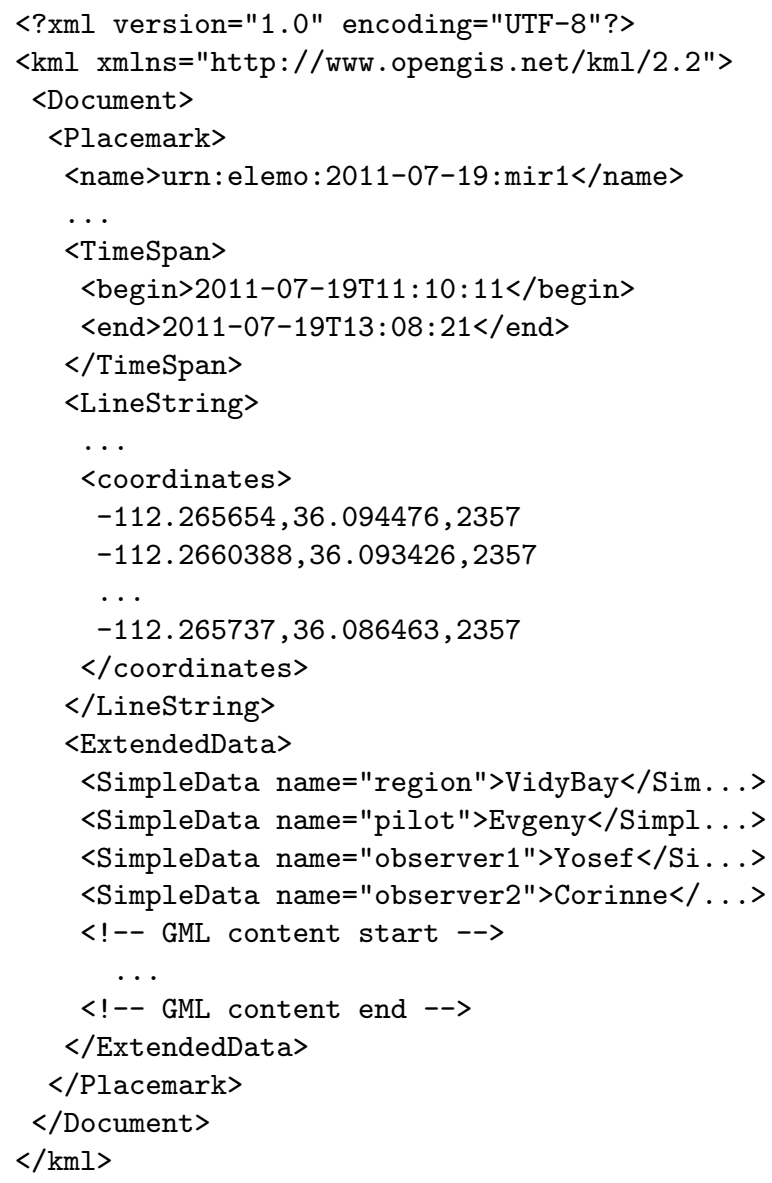

\section{REFERENCES}

Dragicevic, S., 2004. The potential of Web-based GIS. Journal of Geographical Systems 6, pp. 79-81. 10.1007/s10109-004-0133-4.

Fu, P. and Sun, J., 2010. Web GIS: Principles and Applications. Esri Press.

Lake, R., 2007. Transporting gml in kml. Blog post: http: //www . galdosinc.com/archives/308.

OGC® Geography Markup Language (GML) - Extended schemas and encoding rules, 2012. Open Geospatial Consortium, Reference number: 10-129r1. Available: https://portal. opengeospatial .org/files/?artifact_id $=46568$.

OGC® KML, 2008. Open Geospatial Consortium, Version: 2.2.0, Reference number: 07-147r2. Available: http://portal. opengeospatial.org/files/?artifact_id=27810.

OGC® Reference Model, 2011. Open Geospatial Consortium, Reference number: 08-062r7. Available: https://portal. opengeospatial .org/files $/$ ?artifact_id $=47245$.

OGC® SWE Common Data Model Encoding Standard, 2011. Open Geospatial Consortium, Version: 2.0, Reference number:
Table 2: Dense sensor data from the Élémo field campaign encoded using OGC GML Observations-and-Measurements (OM, 2011) and Sensor Web Enablement (SWE, 2011) frameworks and embedded within a KML file, as portrayed in Table 1.

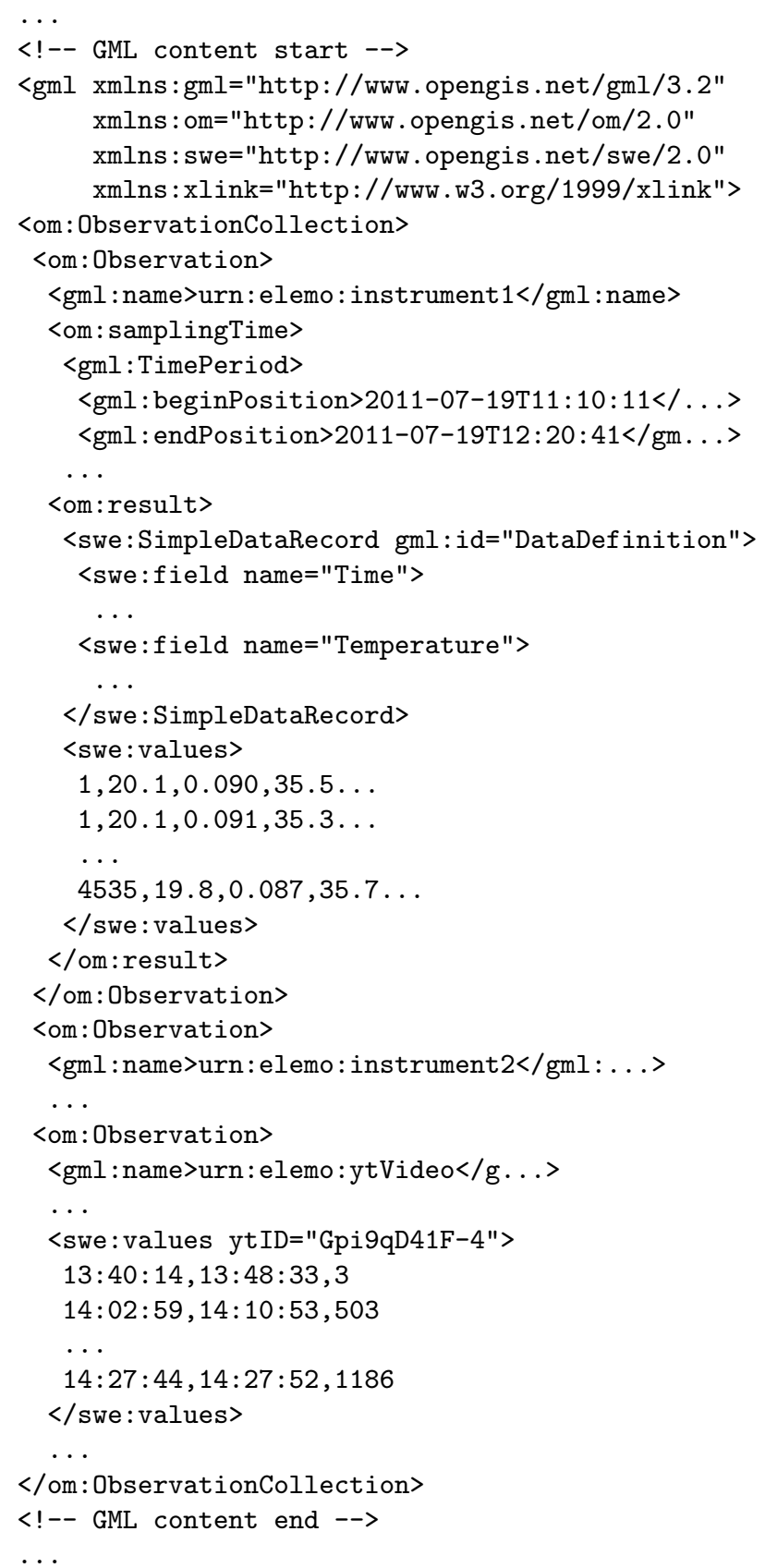

08-094r1. Available: http://portal.opengeospatial.org/ files/?artifact_id=41157. 